\title{
DESEMPENHO DO ENWATBAL PARA AVALIAR A UMIDADE DO SOLO E O USO DA ÁGUA EM UM SISTEMA AGROFLORESTAL ${ }^{1}$
}

\author{
Joaquim Alex Rodrigues Duran² e José Holanda Campelo Júnior ${ }^{3}$
}

\begin{abstract}
RESUMO - O objetivo deste trabalho foi avaliar o desempenho do modelo de simulação ENWATBAL para a estimativa da variação da quantidade de água do solo, em um sistema agroflorestal. Para permitir a validação do modelo, comparou-se a quantidade de água no solo medida com a estimada. O erro-padrão da estimativa indicou variações de 0,02 a $0,1 \mathrm{~m}^{3} \cdot \mathrm{m}^{-3}$ na umidade volumétrica e 8,66 a $22 \mathrm{~mm}$ no armazenamento de água no solo. Os menores erros-padrão foram encontrados nas profundidades entre $0,3-0,6 \mathrm{~m}$, seguidas pelas profundidades de 0,6 - 0,9 e 0,0 - 0,3 m. O índice de concordância variou de 0,51 a 0,93, sendo os maiores valores encontrados na profundidade de 0,3-0,6 m e os menores nas profundidades de $0-0,3$ e 0,3-0,6 m. A eficiência do uso da água na Teca (Tectona grandis L. F.) variou de $1,70 \mathrm{~g} \mathrm{~kg}^{-1}$ a $2,43 \mathrm{~g} \mathrm{~kg}^{-1}$; na mandioca (Manihot esculenta Crantz), variou de 0,13 a 1,29 $\mathrm{g} \mathrm{kg}^{-1}$; e no milho (Zea mays L.), de 0,53 a $0,71 \mathrm{~g} \mathrm{~kg}^{-1}$. Nos consórcios entre os cultivos anuais e as árvores, verificou-se que a Teca consumiu maior quantidade de água que a mandioca e o milho. Verificou-se, também, que a mandioca apresentou menor competição por água quando comparada com o milho, nos respectivos ciclos.
\end{abstract}

Palavras-chave: Simulação, água no solo e sistemas agroflorestais.

\section{ENWATBAL'S PERFORMANCE IN THE EVALUATION OF SOIL MOISTURE AND WATER USE IN AN AGROFORESTRY SYSTEM}

\begin{abstract}
The objective of this work was to evaluate the simulation model ENWATBAL's performance in the estimate of the soil water amount variation in an agroforestry system. The amount of measured soil water was compared with the estimated amount to validate the model. The estimate's standard error showed variations between 0.02 and $0.1 \mathrm{~m}^{3} \mathrm{~m}^{-3}$ for volumetric moisture and 8.66 to $22 \mathrm{~mm}$ for soil water storage. The lesser standard errors of the estimate were found at depths between $0.3-0.6 \mathrm{~m}$, followed by depths of $0.6-0.9$ and $0.0-0.3 \mathrm{~m}$. The agreement index varied from 0.51 to 0.93 , with the highest values being found at the depth of $0.3-0.6 \mathrm{~m}$ and the lowest at the depths of $0-0.3$ and $0.3-0.6 \mathrm{~m}$. Water use efficiency varied from $1.70 \mathrm{~g} \mathrm{~kg}^{-1}$ to $2.43 \mathrm{~g} \mathrm{~kg}^{-1}$, for teak, 0.13 to $1.29 \mathrm{~g} \mathrm{~kg}^{-1}$ for cassava, and 0.53 at $0.71 \mathrm{~g} \mathrm{~kg}^{-1}$ for maize. In the consortium of annual crops and trees, it was verified that teak consumed a greater amount of water than cassava and maize. Cassava presented a lesser competition for water than maize, in the respective cycles.
\end{abstract}

Keywords: Agroforestry, simulation and soil water.

\section{INTRODUÇÃO}

A água é de fundamental importância na produção vegetal, pois sua falta ou excesso afeta, de maneira significativa, o desenvolvimento das plantas. A necessidade de explorá-la e manejá-la eficientemente é uma das tarefas mais importantes do nosso tempo.
O movimento da água em ambientes florestais, seja natural ou de plantações de espécies de crescimento rápido, depende da precipitação, da interceptação da água pelo dossel, do escoamento lateral e em profundidade (drenagem profunda) e da evapotranspiração. Com exceção da precipitação, os demais processos são bastante influenciados pela densidade

\footnotetext{
${ }^{1}$ Recebido em 24.01.2007 e aceito para publicação em 22.08.2008.

${ }^{2}$ Programa de Pós-Graduação em Agricultura Tropical da UniversidadeFederal deMato Grosso(UFMT). E-mail:<joaquimduran@terra.com.br>. ${ }^{3}$ Departamento de Solos e Engenharia Rural da UFMT. E-mail:<campelo@cpd.ufmt.br>.
} 
de plantas, pelo tipo de solo, pelo comportamento fisiológico da planta e pela estrutura e arquitetura do dossel (ALMEIDA e SOARES, 2003). A evapotranspiração depende fundamentalmente da radiação solar, cuja disponibilidade num sistema agroflorestal pode ser modificada pelas árvores (RIGHI et al., 2007).

Para o estabelecimento de um sistema de produção agroflorestal, a validação de um modelo de simulação permite obter, rapidamente, indicações sobre a competição entre as espécies por água e energia, podendo ser aplicado em práticas de manejo que se mostrem promissoras quanto à produção e proteção do solo.

Os modelos de simulação vêm desempenhando papel importante para o entendimento e monitoramento de processos em diversos monocultivos, como o de milho, algodão, soja, arroz, sorgo e girassol (HOOGENBOOM, 2000). No caso de sistemas agroflorestais, o emprego de modelagem tem sido dificultado pela maior complexidade inerente aos sistemas, sobretudo no que diz respeito à sua maior variabilidade espacial (MUETZELFELDT e SINCLAIR, 1993).

O modelo de simulação ENWATBAL foi primeiramente proposto por Lascano et al. (1987) e derivado da combinação de três diferentes modelos: Conservb (LASCANO et al., 1983), Watbal (van BAVEL et al., 1984) e Microweather (CHEN, 1984). A primeira versão foi escrita em Continuous System Modeling Program (CSMP), e com o advento dos computadores PCs foi desenvolvido em linguagem BASIC (EVETT e LASCANO, 2002). O modelo já foi usado para estimar a evapotranspiração (ET) no algodão e sorgo (LASCANO et al., 1987). Campelo Júnior et al. (2002), avaliando o desempenho na previsão da umidade do solo cultivado com uma pastagem de Paspalum atratum Swallen em condições de cerrado, verificaram desempenho satisfatório na determinação do regime de umidade do solo.

Originalmente, o modelo foi concebido para um dossel uniforme, como o existente em monocultivos de culturas agrícolas anuais. No entanto, Oliveira (1994) utilizou o ENWATBAL para estudar a transpiração em uma área cultivada com nove espécies diferentes, incluindo gramíneas rasteiras, arbustos e árvores e introduzindo algumas modificações simples na resistência aerodinâmica e na radiação absorvida, bem como procedeu ao cálculo da transpiração por unidade de superfície do solo para gramíneas e por unidade de superfície foliar para plantas isoladas.

R. Árvore, Viçosa-MG, v.32, n.5, p.879-889, 2008
O objetivo deste trabalho foi avaliar o desempenho do modelo de simulação ENWATBAL para a estimativa da variação da quantidade de água do solo em um sistema agroflorestal constituído por Teca (Tectonia grandis L. F), milho (Zea mays L.) e mandioca (Manihot esculenta Crantz), de modo a permitir a avaliação do uso da água por cada um desses integrantes do sistema.

\section{MATERIAL E MÉTODOS}

O experimento foi instalado em março de 1999 , na Fazenda Experimental da UFMT, no Município de Santo Antônio do Leverger, localizada próxima à sede do referido município, a $15^{\circ} 47^{\prime} 5^{\prime \prime} \mathrm{S}$ de latitude, longitude $56^{\circ} 04^{\prime} \mathrm{W}$ e altitude da estação meteorológica de 140 m acima do nível do mar. A região, denominada Depressão Cuiabana, apresenta clima do tipo Aw, solos litólicos distróficos, concrecionários (Plintossolos), às vezes epicascalhentos, com profundidade média de $0,90 \mathrm{~m}$ (BRASIL, 1982).

O experimento consistiu de três blocos com os seguintes tratamentos: 1 - Cultivo de Teca no espaçamento $3 \times 3 \mathrm{~m}$, em consórcio com milho (Teca 3 x 3 + milho); 2 - Cultivo de Teca no espaçamento $3 \times 3$ m, em monocultivo (Teca $3 \times 3$ ); 3 - Cultivo de Teca no espaçamento $3 \times 3 \mathrm{~m}$, em consórcio com mandioca (Teca 3 × 3 + mandioca) 4 - Cultivo de Teca no espaçamento $6 \times 3 \mathrm{~m}$, em consórcio com a mandioca (Teca 6 × 3 + Mandioca); 5 - Cultivo de Teca no espaçamento $6 \times 3 \mathrm{~m}$, em monocultivo (Teca $6 \times 3$ ); 6 - Cultivo de Teca no espaçamento 6 × 3 m, em consórcio com milho (Teca $6 \times 3+$ milho); 7 - Mandioca em monocultivo; e 8 - Milho em monocultivo.

A Teca foi plantada em março de 1999 e apresentou altura média de $7 \mathrm{~m}$ ao longo do período de observação deste trabalho. A mandioca cv. Liberata foi plantada em 30 de dezembro de 2002, no espaçamento de 0,8 $\mathrm{x} 1 \mathrm{~m}$ em todas as parcelas, e colhida em 22 de dezembro de 2003. O milho variedade BR 106 foi semeado em 31 de dezembro de 2002, no espaçamento de $0,2 \times 0,8$ m, e colhido em 15 de abril de 2003.

Foram realizadas medições de umidade do solo ( $\theta$ ) de acordo com Kiehl (1979), em três faixas de profundidade $(0,0-0,3 \mathrm{~m}, 0,3-0,6 \mathrm{~m}$ e $0,6-0,9$ $\mathrm{m})$, em 14 intervalos de tempo de duração variável, de 6 a 73 dias, de novembro de 2002 a novembro de 2003. 
Foram realizadas medidas de crescimento das plantas em todas as parcelas de todos os tratamentos, ao longo do mesmo período de 12 meses, e sua análise completa foi objeto do trabalho de Brauwers (2004).

Nos dias de medição de umidade do solo, foram realizadas medidas de temperatura do solo e de radiação solar incidente acima e abaixo das copas de Teca, durante todo o período diurno, em uma parcela de Teca em monocultivo no espaçamento $3 \times 3 \mathrm{~m}$, em uma parcela de mandioca em monocultivo, em uma parcela de milho em monocultivo e em uma parcela de Teca cultivada no espaçamento $6 \times 3 \mathrm{~m}$, em consórcio com mandioca. As mesmas medições não foram realizadas em parcelas dos outros tratamentos em função do número de sensores e registradores disponíveis. Essas parcelas foram escolhidas aleatoriamente entre todas as parcelas de todos os tratamentos, pela ordem em que foram sorteadas, descartando-se as repetições até obter todos os monocultivos, os dois espaçamentos da Teca e um consórcio. Os dados obtidos dessas parcelas foram utilizados para verificar a validade do modelo nesse experimento. As simulações foram realizadas em todos os intervalos de coleta de dados de umidade e de temperatura do solo, de modo que os dados do início do intervalo pudessem ser utilizados como dados de entrada daquele período e como dados de validação da simulação do período anterior.

Após a validação do modelo, no mesmo período as simulações foram efetuadas em todos os tratamentos, para estimar a evapotranspiração e a transpiração de cada componente do sistema agroflorestal no experimento e avaliar o uso da água.

Para a simulação dos tratamentos em monocultivo, considerou-se toda a radiação global incidente medida na estação meteorológica, bem como os dados de área foliar e enraizamento próprios de cada espécie.

Nos tratamentos em consórcio, procedeu-se a duas simulações. A primeira foi realizada de modo semelhante ao usado nos monocultivos, sem distinção de espécie, utilizando-se a soma das áreas foliares das duas espécies do consórcio e o enraizamento da espécie arbórea. A segunda foi efetuada no milho ou na mandioca, considerando-se a radiação transmitida pela Teca, a área foliar e o respectivo enraizamento das espécies sombreadas pela Teca.
Os dados meteorológicos necessários foram: radiação solar sobre a cultura $\left(\mathrm{MJm}^{-2} \mathrm{~d}^{-1}\right)$, radiação solar global para a Teca, mandioca em monocultivo e milho em monocultivo e radiação incidente abaixo das copas para as culturas anuais sombreadas, temperatura máxima e mínima diária $\left({ }^{\circ} \mathrm{C}\right)$, temperatura máxima e mínima diária do ponto de orvalho $\left({ }^{\circ} \mathrm{C}\right)$, velocidade do vento $\left(\mathrm{ms}^{-1}\right)$ obtida a $2 \mathrm{~m}$ de altura da superfície e pressão atmosférica $(\mathrm{kPa})$.

A simulação exigiu, ainda, o fornecimento de dados de índice de área foliar $\left(\mathrm{m}^{2} \mathrm{~m}^{-2}\right)$, profundidade do enraizamento $(\mathrm{m})$ e profundidade com maior quantidade de raiz $(m)$.

A área foliar $\left(\mathrm{AF}, \mathrm{m}^{2}\right)$ da Teca, em cada dia, foi obtida por interpolação linear dos valores estimados mensalmente por meio de um método indireto, no qual ela foi calculada em função do volume da copa de cada árvore e da densidade foliar média. Essa densidade foi obtida por um método destrutivo, aplicado mensalmente durante o período de novembro de 2002 a novembro de 2003, em que foram retiradas todas as folhas de três plantas, sorteadas aleatoriamente em um plantio próximo ao experimento, que apresentavam idade, altura e volume de copa semelhantes aos das plantas das parcelas experimentais.

A área foliar da mandioca e do milho foi estimada mensalmente através de medidas não-destrutivas realizadas em três plantas de cada parcela e de relações alométricas determinadas previamente. A interpolação linear dos valores mensais também foi utilizada para obter os valores diários de área foliar da mandioca e do milho.

A profundidade do enraizamento $(\mathrm{m})$ e a profundidade com maior quantidade de raiz $(\mathrm{m})$ foram determinadas em amostras de solo coletadas por tradagem.

Utilizou-se o erro-padrão da estimativa, conforme a equação 1, para comparar a umidade medida e a calculada pelo modelo (ALMEIDA et al., 1999):

$s=\sqrt{\frac{\sum\left(\theta_{m}-\theta_{e}\right)^{2}}{n-2}}$

em que: $\theta=$ umidade volumétrica, $\mathrm{n}=$ número de observações e os subscritos, $\mathrm{m}=$ medida e e $=$ estimada.

R. Árvore, Viçosa-MG, v.32, n.5, p.879-889, 2008 
Além disso, utilizou-se o índice de concordância (d) (WILLMOTT et al., 1985), que expressa a exatidão das estimativas em relação aos valores observados, variando de zero, que indica nulidade, a 1, que indica perfeita exatidão. O índice d é determinado da seguinte forma:

$$
d=1-\left[\frac{\sum\left(\theta_{e i}-\theta_{m i}\right)^{2}}{\sum\left(\left|\theta_{e i}-\bar{\theta}_{m}\right|+\left|\theta_{m i}-\bar{\theta}_{m}\right|\right)^{2}}\right]
$$

em que: $\bar{\theta}=$ média dos valores medidos de umidade do solo e $\mathrm{i}=$ número de eventos (dias).

Os dados estimados e observados foram, ainda, comparados através de uma análise de regressão linear simples.

Verificou-se a eficiência do uso da água da Teca, milho e mandioca. Na Teca foi utilizada a produção de matéria seca estimada de madeira. Para a mandioca e o milho, usou-se a produção de matéria seca total.

Para estimar a matéria seca de madeira produzida, considerou-se a densidade da madeira de $0,55 \mathrm{~g} \mathrm{~cm}^{-}$ ${ }^{3}$ (FRANCIS e LOWE, 2000), com teor de matéria seca de $18,72 \%$ (RAJAN et al., 2000). O volume de madeira na Teca, no ano de 2003, foi determinado pela cubagem de Smalian, descrito por Finger (1992). Para isso, avaliaram-se o Diâmetro à Altura do Solo (DAS), Diâmetro à Altura do Peito (DAP $=\mathrm{h}=1,3 \mathrm{~m}$ ) e Altura da Planta (AP), no intervalo de 120 dias.

Na mandioca e no milho, a massa seca foi determinada no final dos respectivos ciclos, no Laboratório do Núcleo de Tecnologia em Armazenagem (NTA/FAMEV), em estufa a $72^{\circ} \mathrm{C}$, até as amostras adquirirem peso constante.

A eficiência do uso da água da produtividade (EUA) foi determinada pela equação 3 , que expressa numericamente a razão global entre a produção de matéria seca e o consumo de água (LARCHER, 2000).

$E U A=\frac{\text { massa seca produzida }}{\text { consumo de água }}$

O consumo de água foi considerado como a transpiração total de cada espécie vegetal, durante os ciclos nas culturas anuais e durante o ano na Teca, sendo calculada diariamente em cada espécie, pelo modelo de simulação ENWATBAL.

R. Árvore, Viçosa-MG, v.32, n.5, p.879-889, 2008

\section{RESULTADOS E DISCUSSÕES}

Na comparação entre a umidade medida e a estimada pelo modelo ENWATBAL o erro-padrão mostrou variações de 0,02 a $0,1 \mathrm{~m}^{3} \mathrm{~m}^{-3}$. Os menores erros-padrão foram encontrados nas profundidades de 0,3 - 0,6 m, seguidas pelas profundidades de 0,6 - 0,9 e 0,0 - 0,3 $\mathrm{m}$. O índice de concordância (d) apresentou resultados semelhantes, pois os maiores valores foram encontrados na profundidade de 0,3 - 0,6 $\mathrm{m}$ e os menores, de 0 0,3 e 0,3 - 0,6 m (Figuras 1 a 4).
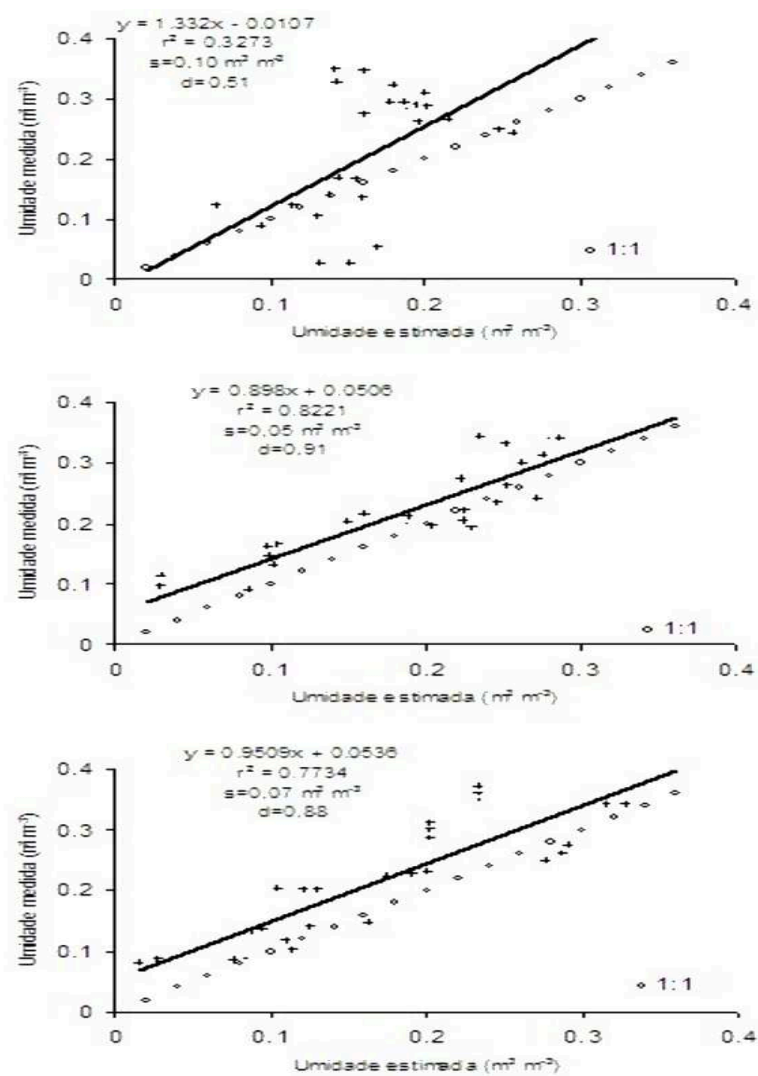

Figura 1 - Relação entre a umidade volumétrica medida e a estimada pelo modelo Enwatbal, em monocultivo de Teca no espaçamento $3 \times 3 \mathrm{~m}$, nas camadas de solo de 0 a $0,3 \mathrm{~m}$ (figura superior), de $0,3 \mathrm{a}$ $0,6 \mathrm{~m}$ (figura intermediária) e de 0,6 a $0,9 \mathrm{~m}$ de profundidade (figura inferior), em Santo Antônio do Leverger, MT, de novembro de 2002 a novembro de 2003.

Figure 1-Relation between the volumetric moisture measured and estimated by the Enwatbal model in teak monoculture, on $3 \times 3$ grid, at O-O.3 m (top figure), in soil layers of 0.3-0.6 m (mean figure), and 0.6$0.9 \mathrm{~m}$ (bottom figure), at Santo Antônio do LevergerMT, November 2002-November 2003. 


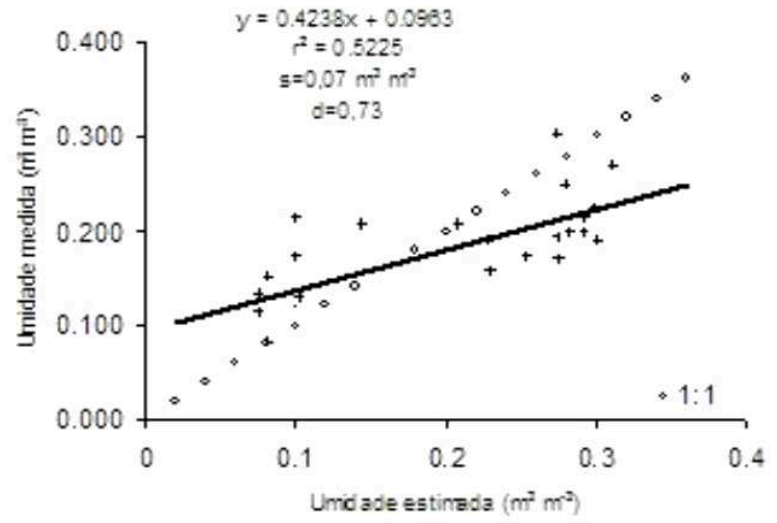

Figura 2 - Relação entre a umidade volumétrica medida e a estimada pelo modelo Enwatbal, em consórcio de mandioca e Teca no espaçamento $6 \times 3 \mathrm{~m}$, nas camadas de solo de 0 a 0,3 m, em Santo Antônio do Leverger, MT, de novembro de 2002 a novembro de 2003.

Figure 2-Relation between the volumetric moisture measured and estimated by the Enwatbal model in cassava and teak consortium, on $6 \times 3$ grid, in soil layers of of O-O.3 m, in Santo Antônio do Leverger-MT, November 2002-November 2003.

Na Tabela 1, apresenta-se o resultado das análises estatísticas do armazenamento de água no solo, na profundidade de 0 - 0,9 m, estimadas pelo modelo de simulação ENWATBAL, comparados com observações de campo. A significância dos coeficientes de correlação entre os valores medidos e os estimados de umidade no solo foi inferior a $1 \%$, em todas as parcelas usadas na validação.

Ao verificar o armazenamento de água no solo (ARM) medido em relação ao estimado, na Teca $3 \mathrm{x}$ 3 , à profundidade de 0 - 0,9 m (Figura 5), observouse que os valores estimados apresentaram tendência a subestimar o ARM a partir do mês de maio. No mês de setembro, o ARM estimado foi zero, enquanto o observado, $17 \mathrm{~mm}$. Essa tendência foi provocada pelos valores de umidade volumétrica medida, que se mostraram superiores aos medidos nas três faixas de profundidade, como se pode ver pela Figura 1, em que as retas de regressão ficaram acima da linha 1:1. Apesar disso, através do modelo, foi possível estimar satisfatoriamente o ARM, na Teca 3 x 3 e na profundidade $0-0,9$ m, cujos valores estimados e medidos tiveram correlação positiva de 0,95 e coeficiente de determinação $\left(\mathrm{R}^{2}\right)$ de 0,90 . $\mathrm{O}$ erro-padrão da estimativa foi de $8,7 \mathrm{~mm}$ e o índice de concordância, 0,97 .
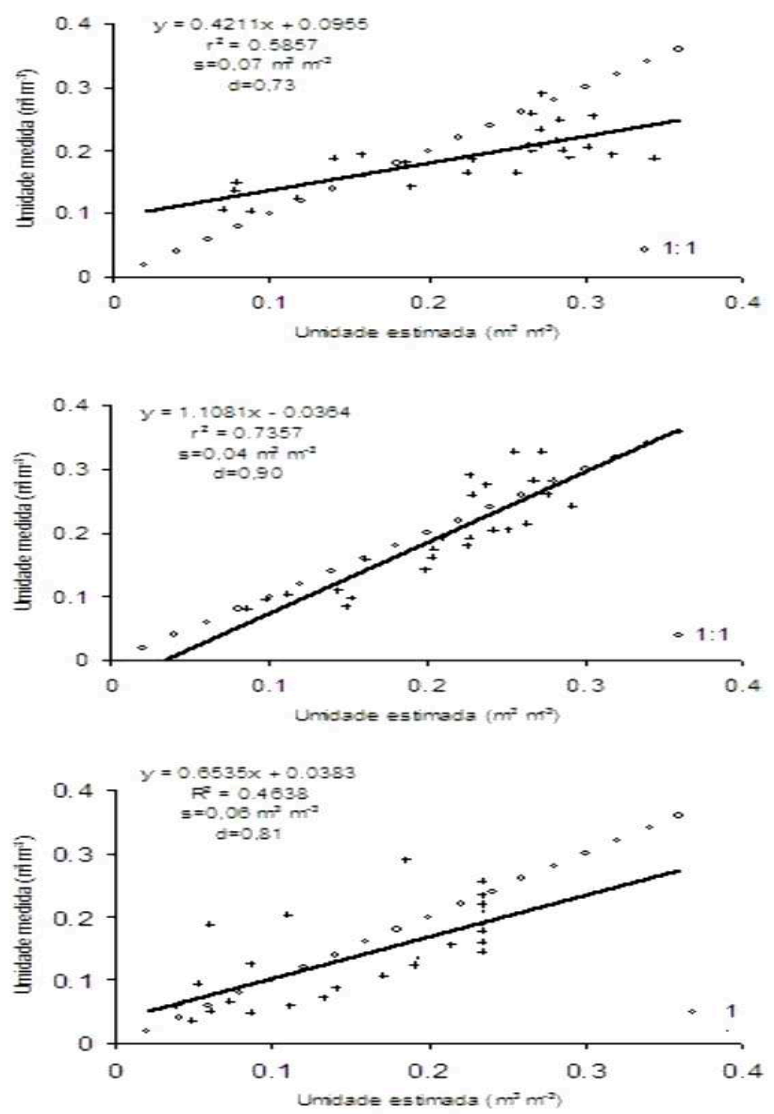

Figura 3 - Relação entre a umidade volumétrica medida e a estimada pelo modelo Enwatbal, em monocultivo de mandioca nas camadas de solo de 0 a $0,3 \mathrm{~m}$ (figura superior), de $0,3 \mathrm{a} 0,6 \mathrm{~m}$ (figura intermediária) e de 0,6 a $0,9 \mathrm{~m}$ de profundidade (figura inferior), em Santo Antônio do Leverger, MT, de novembro de 2002 a novembro de 2003 .

Figure 3-Relation between the volumetric moisture measured and estimated by the Enwatbal model in cassava monoculture, in soil layers of O-0.3 m (top figure), 0.3-0.6 m (mean figure), and 0.6-0.9 m depth (bottom figure), at Santo Antônio do LevergerMT, November 2002-November 2003.

Na Teca cultivada no espaçamento 6 x 3 em consórcio com a mandioca, os valores medidos e estimados de armazenamento até a profundidade de 0,3 m apresentaram coeficiente de determinação de 0,85 , correlação positiva de 0,92 , erro-padrão de 7,2 mm e índice de concordância de 0,88. Alguns valores de armazenamento de água foram superestimados em períodos com maior ARM, mas o modelo não apresentou tendência definida para subestimar ou superestimar os valores observados (Figura 6).

R. Árvore, Viçosa-MG, v.32, n.5, p.879-889, 2008 

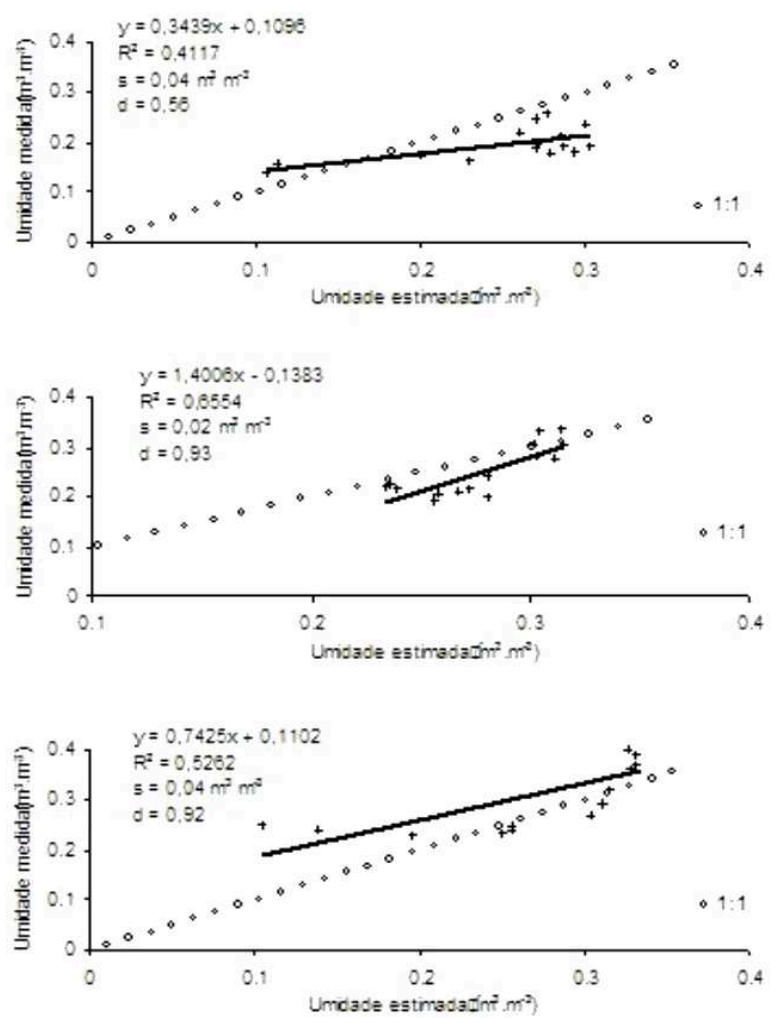

Figura 4 - Relação entre a umidade volumétrica medida e a estimada pelo modelo Enwatbal, em monocultivo de milho nas camadas de solo de 0 a $0,3 \mathrm{~m}$ (figura superior), de 0,3 a $0,6 \mathrm{~m}$ (figura intermediária) e de 0,6 a $0,9 \mathrm{~m}$ de profundidade (figura inferior), em Santo Antônio do Leverger, MT, de novembro de 2002 a novembro de 2003.

Figure 4-Relation between the volumetric moisture measured and estimated by the Enwatbal model in maize monoculture, in soil layers of 0-0.3 m (top figure), 0.3-0.6 m (mean figure), and 0.6-0.9 m depth (bottom figure), in Santo Antônio do Leverger-MT, November 2002-November 2003.
Na mandioca em monocultivo, o modelo estimou o ARM na profundidade de 0 - $0,9 \mathrm{~m}$, com correlação positiva de 0,84 e coeficiente de determinação de 0,71, erro-padrão de $14 \mathrm{~mm}$ e índice de concordância de 0,9. Os valores de ARM foram, na maioria das comparações, superestimados pelo modelo. Entretanto, os valores de umidade volumétrica na profundidade de 0 - 0,3 m (Figura 3) não apresentaram essa tendência. Possivelmente esse fato esteja relacionado ao procedimento adotado no modelo para estimar a absorção de água como diretamente proporcional à maior densidade de raízes. No caso da mandioca, o procedimento pode não ser adequado, acarretando estimativas de retiradas de água dessa profundidade maiores do que os valores reais. A compensação representada pelas superestimativas de umidade nas profundidades maiores pode levar ao cálculo mais preciso da transpiração.

No milho, o ARM estimado apresentou correlação positiva de 0,83 quando comparado com o que foi medido. O coeficiente de determinação $\left(r^{2}\right)$ foi de 0,68. Apresentou erro-padrão da estimativa de 22,4 mm e índice de concordância de 0,89 . O modelo foi subestimado na maioria das comparações, porém verificou-se superestimativa quando o ARM foi menor que $120 \mathrm{~mm}$.

É necessário ressaltar que valores instantâneos de armazenamento de água no solo (ARM) podem ser maiores que a capacidade de água disponível (CAD). Essa diferença representa o excedente hídrico, que é a quantidade de água que sobra no período chuvoso e se perde por drenagem profunda (LIMA e LEOPOLDO, 2000; PEREIRA et al, 2002).

Tabela 1 - Coeficiente de determinação $\left(r^{2}\right)$, erro-padrão da estimativa (s) e índice de concordância (d) entre valores de armazenamento de água no solo medidos e estimados pelo modelo Enwatbal, em um sistema agroflorestal, em Santo Antônio do Leverger, MT, de novembro de 2002 a novembro de 2003

Table 1 - Coefficient of determination $\left(r^{2}\right)$, estimate standard error $(s)$ and index of agreement $(d)$ between soil water storage values measured and estimated by the Enwatbal model in an agroforestry system in Santo Antônio do LevergerMT, November 2002-November 2003

\begin{tabular}{|c|c|c|c|}
\hline Tratamento & $\mathrm{r}^{2}$ & smm & $\mathrm{D}$ \\
\hline Monocultivo de Teca no espaçamento $3 \times 3 \mathrm{~m}$ & 0,90 & 8,7 & 0,97 \\
\hline Monocultivo de Teca no espaçamento $6 \times 3 \mathrm{~m}$ & 0,85 & 7,2 & 0,88 \\
\hline Monocultivo de Mandioca & 0,71 & 14,0 & 0,90 \\
\hline Monocultivo de Milho & 0,68 & 22,4 & 0,89 \\
\hline Consórcio de Mandioca e Teça no espaçamento $6 \times 3 \mathrm{~m}$ & 0,52 & 0,07 & 0,73 \\
\hline
\end{tabular}

R. Árvore, Viçosa-MG, v.32, n.5, p.879-889, 2008 


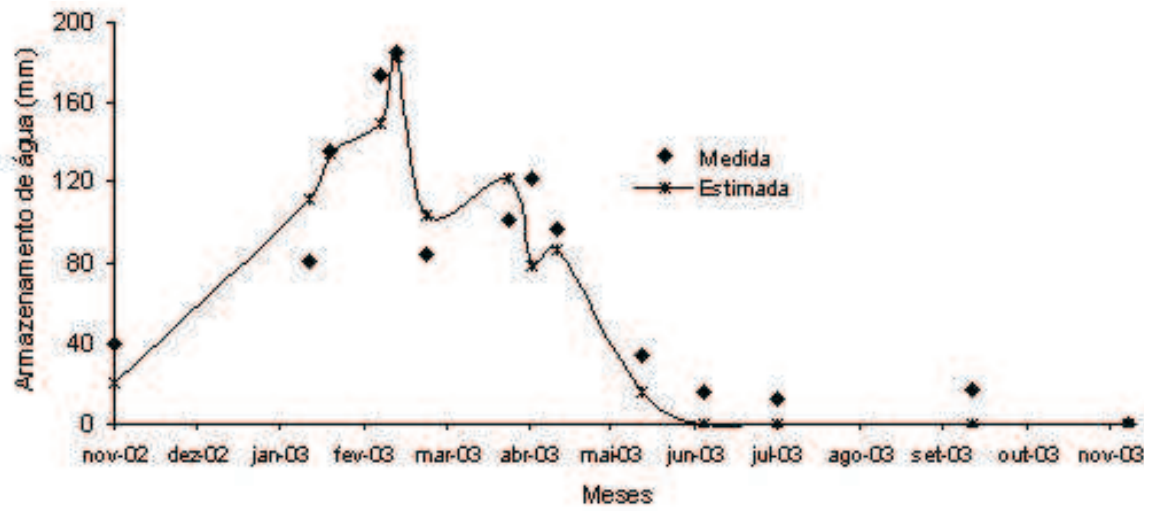

Figura 5 - Comparação entre o armazenamento de água no solo medido e o estimado pelo modelo Enwatbal, em monocultivo de Teca no espaçamento 3 × 3 m, na camada de solo de 0 a 0,9 m de profundidade, em Santo Antônio do Leverger, MT, de novembro de 2002 a novembro de 2003.

Figure 5 - Comparison between soil water storage measured and estimated by the Enwatbal model, in teak monoculture, on $3 \times 3$ grid, in soil layer of 0 to $0.9 \mathrm{~m}$ depth, in Santo Antônio do Leverger-MT, November 2002-November 2003.

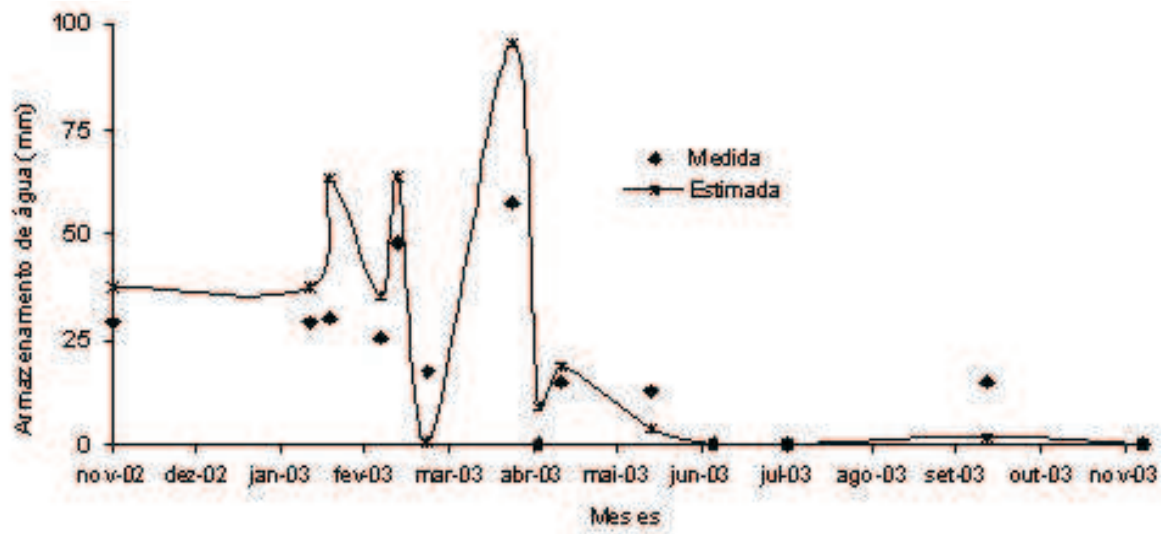

Figura 6 - Comparação entre o armazenamento de água no solo medido e o estimado pelo modelo Enwatbal, em consórcio de mandioca e Teca no espaçamento 6 × 3 m, na camada de solo de 0 a 0,3 m de profundidade, em Santo Antônio do Leverger, MT, de novembro de 2002 a novembro de 2003.

Figure 6-Comparison between soil water storage measured and estimated by the Enwatbal model in cassava and teak consortium, on $6 \times 3 \mathrm{grid}$, in soil layer of 0 to $0.9 \mathrm{~m}$ depth in Santo Antônio do Leverger-MT, November 2002November 2003.

Algumas diferenças nos resultados observados e estimados podem estar relacionadas às medidas de umidade volumétrica, como também às relações empíricas, necessárias ao cálculo do fluxo de água no solo e na transpiração das plantas, uma vez que essas relações interferem, de forma significativa, no cálculo da quantidade de água no solo. O uso de modelo matemático para estimativa de uso da água é prática que facilita o estudo do balanço da água na camada do solo que abriga o sistema radicular. Almeida e Soares (2003) desenvolveram o modelo UAPE (Uso da Água em
Plantações de Eucalipto) e obtiveram, em geral, concordância excelente entre os valores medidos e estimados, sendo de grande utilidade no estudo da comparação do uso da água em plantio de eucalipto (E. grandis).

Através do modelo ENWATBAL foi possível observar as variações da umidade no decorrer do ano hidrológico. Os coeficientes de correlação entre a umidade volumétrica estimada e medida ficaram abaixo dos encontrados por Campelo Júnior et al. (2002) na profundidade $0-0,3 \mathrm{~m}$, porém foram semelhantes nas 
outras profundidades. Os valores estimados apresentaram diferença média de $0,06 \mathrm{~m}^{3} \mathrm{~m}^{-3}$ dos valores medidos. Já os índices de concordância foram superiores aos encontrados por Stone et al. (2002), que avaliaram o desempenho do Sistema de Suporte a Decisão Agrícola (SISDA 2.0).

Na Teca, observou-se que a evapotranspiração estimada pelo modelo foi praticamente igual nos tratamentos, porém não se verificou o mesmo quanto à transpiração (Tabela 2), que foi em média $1,56 \mathrm{~mm}$ $\mathrm{d}^{-1}$ no espaçamento $3 \times 3$, sendo de $1,9 \mathrm{~mm} \mathrm{~d}^{-1}$ no espaçamento $6 \times 3$. Conseqüentemente, a Eficiência do Uso da Água (EUA) na Teca variou de $1,70 \mathrm{~g} \mathrm{~kg}^{-}$ ${ }^{1}$ a $2,43 \mathrm{~g} \mathrm{~kg}^{-1}$ (Tabela 2). Os valores de EUA no espaçamento $6 \times 3$ foram superiores ao espaçamento $3 \times 3$, onde se observaram os maiores valores de EUA nos sistemas Teca $6 \times 3+$ milho e Teca $6 \times 3$. No espaçamento $3 \times 3$, o sistema Teca $3 \times 3+$ milho apresentou maior EUA. Os maiores valores da EUA no espaçamento $6 \times 3$ foram atribuídos à densidade de plantas nesses sistemas, resultando em menor competição por água e maior interceptação de luz.
Os valores de EUA no espaçamento 3 x 3 foram semelhantes aos apresentados por Larcher (2000) para árvores tropicais cultivadas, em que se verificou que a EUA variou de 1 a $2 \mathrm{~g} \mathrm{~kg}^{-1}$. Entretanto, os valores de EUA no espaçamento 6 x 3 estiveram acima desse intervalo. A EUA da Teca no espaçamento $6 \times 3$ foi semelhante à observada por Damiano et al. (2002) em uma plantação de Eucalyptus dunnii, que obtiveram EUA de 2,74 $\mathrm{g} \mathrm{kg}^{-1}$.

Na mandioca, a EUA variou no experimento de 0,13 a $1,29 \mathrm{~g} \mathrm{~kg}^{-1}$. Foram destacados os maiores valores no monocultivo, seguido pela cultura consorciado com a Teca, no espaçamento 6 × 3, e os menores valores foram observados no consórcio com a Teca no espaçamento $3 \times 3$ (Tabela 3). Os valores da EUA na mandioca em monocultivo foram próximos aos encontrados por Yao et al. (1988), ao estudarem a EUA na mandioca em condições de sequeiro e irrigada. Observaram que a EUA variou de 0,77 a 2,26 $\mathrm{g} \mathrm{kg}^{-1}$ em condições de sequeiro e de 1,76 a $2,71 \mathrm{~g} \mathrm{~kg}^{-1}$ na mandioca irrigada.

Tabela 2 - Evapotranspiração, transpiração e eficiência no uso da água da Teca no ano de 2003, em sistema agroflorestal. Santo Antônio do Leverger, MT

Table 2 - Evapotranspiration, transpiration, and water use efficiency of teak in 2003, in an agroforestry system, Santo Antônio do Leverger-MT, November 2002-November 2003

\begin{tabular}{|c|c|c|c|c|}
\hline \multirow[t]{2}{*}{ Tratamento } & \multirow{2}{*}{$\frac{\text { Período }}{\text { (dias) }}$} & Evapotranspiração & Transpiração & \multirow{2}{*}{$\frac{\mathrm{EUA}}{\left(\mathrm{g} \mathrm{kg}^{-1}\right)}$} \\
\hline & & \multicolumn{2}{|c|}{$(\mathrm{mm})$} & \\
\hline \multirow[t]{2}{*}{ Consórcio de Milho e Teca no espaçamento $3 \times 3 \mathrm{~m}$} & $334^{\mathrm{a}}$ & 1168 & 528 & 1,81 \\
\hline & $108^{\mathrm{b}}$ & 569 & 335 & $*$ \\
\hline Monocultivo de Teca no espaçamento $3 \times 3 \mathrm{~m}$ & $334^{\mathrm{a}}$ & 1172 & 474 & 1,70 \\
\hline Consórcio de Mandioca e Teca no espaçamento $3 \times 3 \mathrm{~m}$ & $334^{\mathrm{a}}$ & 1168 & 394 & 1,78 \\
\hline Consórcio de Mandioca e Teca no espaçamento $6 \times 3 \mathrm{~m}$ & $334^{\mathrm{a}}$ & 1177 & 468 & 2,37 \\
\hline Monocultivo de Teca no espaçamento $6 \times 3 \mathrm{~m}$ & $334^{\mathrm{a}}$ & 1172 & 370 & 2,12 \\
\hline \multirow{2}{*}{ Consórcio de Milho e Teca no espaçamento 6x3 m } & $334^{a}$ & 1173 & 410 & 2,43 \\
\hline & $108^{b}$ & 562 & 263 & $*$ \\
\hline
\end{tabular}

${ }^{a}$ período total; ${ }^{\mathrm{b}}$ período de cultivo do milho; * matéria seca não determinada no período.

Tabela 3 - Evapotranspiração, transpiração e eficiência no uso da água da mandioca em monocultivo e em sistema agroflorestal, em Santo Antônio do Leverger, MT, de novembro de 2002 a novembro de 2003

Table 3 - Evapotranspiration, transpiration and water use efficiency of cassava monoculture and agroforestry system, in Santo Antonio do Leverger-MT, November 2002 -November 2003

\begin{tabular}{|c|c|c|c|}
\hline \multirow[t]{2}{*}{ Tratamento } & Evapotranspiração & Transpiração & \multirow{2}{*}{$\frac{\text { EUA }}{\mathrm{g} \mathrm{kg}^{-1}}$} \\
\hline & \multicolumn{2}{|c|}{$\mathrm{mm}$} & \\
\hline Consórcio de Mandioca e Teca no espaçamento $3 \times 3 \mathrm{~m}$ & 1017 & 41 & 0,13 \\
\hline Consórcio de Mandioca e Teca no espaçamento $6 \times 3 \mathrm{~m}$ & 1008 & 58 & 0,35 \\
\hline Monocultivo de mandioca & 1086 & 169 & 1,29 \\
\hline
\end{tabular}

R. Árvore, Viçosa-MG, v.32, n.5, p.879-889, 2008 
Tabela 4 - Evapotranspiração, transpiração e média da eficiência do uso da água no milho em monocultivo e em sistema agroflorestal, em Santo Antônio do Leverger, MT, de novembro de 2002 a novembro de 2003

Table 4 - Evapotranspiration, transpiration and water use efficiency average in maize in monoculture and agroforestry system, in Santo Antonio do Leverger-MT, November 2002-November 2003

\begin{tabular}{|c|c|c|c|}
\hline \multirow[t]{2}{*}{ Tratamento } & Evapotranspiração & Transpiração & \multirow{2}{*}{$\frac{\text { EUA }}{\mathrm{g} \mathrm{kg}^{-1}}$} \\
\hline & \multicolumn{2}{|c|}{$\mathrm{mm}$} & \\
\hline Consórcio de Milho e Teca no espaçamento $3 \times 3 \mathrm{~m}$ & 424 & 84 & 0,53 \\
\hline Consórcio de Milho e Teca no espaçamento $6 \times 3 \mathrm{~m}$ & 463 & 126 & 0,56 \\
\hline Monocultivo de milho & 499 & 126 & 0,71 \\
\hline
\end{tabular}

No milho, observou-se variação da EUA de 0,53 a $0,71 \mathrm{~g} \mathrm{~kg}^{-1}$ (Tabela 4). O monocultivo se apresentou mais eficiente quanto ao uso da água, seguido pelo consórcio com a Teca no espaçamento 6 × 3 e $3 \times$ 3 . Todos os valores observados ficaram abaixo dos encontrados por Sharratt e Gesch (2002), que observaram variações de EUA de 1,05 a $2,0 \mathrm{~g} \mathrm{~kg}^{-}$ ${ }^{1}$. Kang et al. (2002), ao estudarem a EUA em milho em diferentes níveis de irrigação, observaram valores da EUA de 7,1 a 13,96 $\mathrm{g} \mathrm{kg}^{-1}$.

Comparando os valores da coluna de transpiração nas Tabelas 2, 3 e 4 nos mesmos tratamentos em que houve consórcio, verificou-se que, nos tratamentos com o consórcio entre os cultivos anuais e árvores, a Teca consumiu maior quantidade de água do que a mandioca e o milho.

O consumo de água pela mandioca consorciada com a Teca no espaçamento $3 \times 3$ foi de $9,4 \%$ do consumo total de água do consórcio, e no consórcio com a Teca no espaçamento 6 × 3 esse consumo foi de $11 \%$. Nos dois tratamentos em que foi consorciada, a mandioca consumiu menos de $35 \%$ da água consumida quando constituiu monocultivo (Tabelas 2 e 3 ).

Nos consórcios, o milho apresentou maior competição por água com a Teca do que a mandioca. Observou-se que o milho cultivado em consórcio com a Teca, no espaçamento 3 × 3 , consumiu cerca de $20 \%$ da água consumida pela Teca no período de 108 dias (correspondentes ao ciclo do milho). No consórcio com a Teca no espaçamento 6 × 3, ele consumiu o equivalente a $32 \%$ da transpiração da Teca (Tabelas 2 e 4 ).

Assim, verificou-se que a mandioca apresenta menor competição por água quando comparada com o milho, dentro dos respectivos ciclos, porém deve-se ressaltar que o período de observação da mandioca foi de 334 dias, enquanto o do milho foi de 108 dias.

\section{CONCLUSÕES}

O modelo ENWATBAL pode subestimar ou superestimar a umidade volumétrica nas camadas do solo, mas é eficaz na estimativa do armazenamento de água no perfil.

A Teca cultivada no espaçamento $6 \times 3$ é mais eficiente que no espaçamento $3 \times 3$, quanto ao uso da água.

Em monocultivo, a mandioca e o milho são mais eficientes no uso da água que em consórcio.

A mandioca em monocultivo é mais eficiente que o milho quanto ao uso da água.

Em consórcio com a Teca, o milho é mais eficiente do que a mandioca, quanto ao uso da água.

A Teca consome maior quantidade de água que o milho e a mandioca.

O milho apresenta maior competição pela água com a Teca, em comparação com a mandioca.

\section{REFERÊNCIAS}

ALMEIDA, H. A.; KLAR, A. E.; VILA NOVA, N. A. Comparação de dados de evapotranspiração de referência estimados por diferentes métodos. IRRIGA, v.4, n.2, 1999, p.105-118.

ALMEIDA, A. C.; SOARES, J. V. Comparação entre o uso de água em plantações de Eucalyptus grandis e floresta ombrófila densa (mata atlântica) na costa leste do Brasil. Revista Árvore, v.27, n.2, p.159-170, 2003.

BRASIL. Ministério das Minas e Energia. Departamento Nacional da Produção Mineral. Projeto RADAMBRASIL. Folha SD.21 Cuiabá: geologia, geomorfologia, pedologia, vegetação, uso potencial da terra. Rio de Janeiro: 1982. 444p. (Levantamento de Recursos Naturais, v.12).

R. Árvore, Viçosa-MG, v.32, n.5, p.879-889, 2008 
BRAUWERS, L. R. Interceptação da radiação solar, crescimento e produção dos componentes de um sistema agroflorestal. 2004. 62f. Dissertação (Mestrado em Agricultura Tropical) - Universidade Federal de Mato Grosso, Cuiabá, 2004.

CAMPELO JÚNIOR, J. H.; MEDEIROS, R. A.; BRAUWERS, L. R. Simulação do balanço hídrico do solo em pastagem sob condições de cerrado. In: REUNIÃO BRASILEIRA DE MANEJO E CONSERVAÇÃO DO SOLO E DA ÁGUA, 14., 2002, Cuiabá. Anais... Cuiabá: Universidade Federal de Mato Grosso, 2002. p.148.

CHEN, J. Mathematical analysis and simulation of crop micrometeorology. Thesis (Ph. D.). Wageningen: Agricultural Universty, 1984.

DAMIANO, F. et al. Consumo de água y crecimiento del Eucalyptus dunnii maiden em Santa Fé, Argentina. Misiones, Argentina: INTAFCF-MEYRNRYT-Eldorado. In: Novenas Jornadas Técnicas Forestales, 2002. Disponível em: <http://factor.unan.edu.ar/silvman/ dunnidamiano.pdf.> Acesso em: 12 de fev. 2004.

EVETT, S. R.; LASCANO, R. J. ENWATBAL.BAS: a mechanistic evapo-transpiration model written in compiled basic. Agronomy Journal, v.85, n.3, p.763-772, 1993. Disponível em: http:// www.cprls.ars.usda.gov/programs.htm> Acesso em: 10 de nov. 2002.

FINGER, C. A. G. Fundamentos de biometria florestal. Santa Maria: Universidade Federal de Santa Maria/CEPEF/ FATEC, 1992. 220p.

FRANCIS, J. K.; LOWE, C. A. Bioecología de arboles nativos y exóticos de Puerto Rico y las Indias Occidentales. Rio Piedras: USDA-Instituto Insternacional de Dasononomía Tropical, 2000. p.524-540.

HOOGENBOOM, G. Contribution of agrometeoroly to the simulation of crop production and its aplication. Amsterdam: Agricultural and Forest Meteoroly, v.103, n.1-2, p.137-157, 2000.
KANG, S. et al. Simulation of winter wheat yield and water use efficiency on the loess plateau of China using WAVES. In: KANG, S. et al. Regional water and soil assessment for managing sustaintable agriculture in China and Australia. Australia: 2002. p.95-104.

KIEHL, E. J. Manual de edafologia: relações solo-planta. São Paulo: Ceres, 1979. p.191-213.

LARCHER, W. Ecofisiologia vegetal. São Carlos: Rima, 2000. p.231-294.

LASCANO, R. J.; van BAVEL, C. H. M. Experimental verification of a model to predict soil moisture and temperature profiles. Soil Science Society American Journal, v.47, p.441-448, 1983.

LASCANO, R. J. et al. Energy and water balance of a space crop: simulation and measured soil and crop evaporation. Soil Science Society American Journal, v.51, p.1113-1121, 1987.

LIMA, P. R. A.; LEOPOLDO, P. R. Quantificação de componentes hidrológicos de uma mata ciliar, através do modelo de balanço de massas.

Revista Árvore, v.24, n.3, p.241-252, 2000.

MUETZELFELDT, R. I.; SINCLAIR, F. L.

Ecological modelling of agroforestry systems. Agroforestry Abstracts, v.6, p.207-247, 1993.

OLIVEIRA, R. F. Water and energy fluxes in an urban landscape. 1994. 137f. Thesis Texas: A \& M University, 1994.

PEREIRA, A. R.; ANGELOCCI, L. R.; SENTELHAS, P. C. Agrometeorologia: fundamentos e aplicações práticas. Guaíba: Agropecuária, 2002. p.251-259.

RAJAN, S. K.; REDDY, B. J. D.; BAGYARAJ, D. J. Screening of arbuscular mycorrhizal fungi for their symbiotic effiency with Tectonia grandis. Forest Ecology and Management, v. 126, n.1, p.91-95, 2000. 
RIGHI, C. A. et al. Measurement and simulation of solar radiation availability in relation to the growth of coffee plants in an agroforestry system with rubber trees.

Revista Árvore, v.31, n.2, p.195-207, 2007.

SHARRATT, B. S.; GESCH, R. W. Rooting characteristics and water requirements of Cuphea. In: JANICK,J. \& WHIPKEY, A. (eds.) Trends in new crops and new uses. Alexandria: 2002. p.216-218.

STONE, L. F., MOREIRA, J.A. A.; SILVA, J. G.

Desempenho do modelo computacional SISDA 2.0 no manejo da irrigação do feijoeiro em condições de plantio direto e preparo convencional do solo.

Engenharia Agrícola, v.22, n.3, p.304-311, 2002. van BAVEL, C. H. M.; LASCANO, R. J.;

STROOSNIJDER, L. Test and analysis of model of water use by sorghum. Soil Science, v.137, n.6, p.443-456, 1984.

WILLMOTT, C. J. et al. LEGATES, Statistics for the evaluation and comparision of models.

Journal of Geophysical Research, v.90, n.C12, p.8995-9005, 1985.

YAO, N. R. et al. Effect of drought on leaf development and dry matter production of the cassava (Manihot esculeta Crantz). Plant

Tropical Agricultural, 1988, p.84-88. 
